\title{
Pitfalls and Successes of Building a Dedicated Electron Microscopy Facility from Scratch
}

\section{Eric Hanssen}

The University of Melbourne, Melbourne, Victoria, Australia

Most biological electron microscopy platforms directors/managers/heads are biologists that have specialized in electron microscopy and with increasing pressure in terms of personal or lack thereof have slowly converted to human resource manager, accountant, student counsellor and if time permits have maintained a research active part in their schedule. Not many have the opportunity of adding one more skillset to their quiver: architect or at least Wannabe architect.

In an era where electron microscopy has become a staple of science and where most universities and research institutions are heavily investing in high end electron microscopes, especially in the cryo electron microscopy field, the need for new facilities accommodating such large pieces of equipment has become ubiquitous. After 9 years of lobbying, the University of Melbourne in Australia, like others, has decided to create a purpose build electron microscopy structure to house an ever expanding stable of tools.

The costing of the concept design was based on a rough drawing relying only on surface cost of each different space, teaching space, offices, wet laboratory, low requirement tools (eg. optical microscopes), high requirements tools (eg. electron microscopes) and plant space. The rough nature of the original drawing led to subsequent fund raising exercises to allow for a more complex build and refined design. The original draft was enhanced with more professional ideas and engineering solutions but the original basic philosophies were included, bright and open volumes for operators and staffs, large space for tools, easy workflows.

Key features were included in the plans in order to make the building as environmentally friendly as the technical specification of the tools required and the budget allowed. But for a very large concrete base slabs only timber, locally sourced, metal frames and glass was used for the construction of the three level edifice. All vertical structures are made of steel and all horizontal made of laminated timber including the ceiling of microscope rooms. The standard concrete base for each floor was also replaced with cross laminated timber. The Key feature in the design allowed for fewer framing requirements and a lighter building.

This work will go thru the milestones of four years of design and construction, going through the concepts original concept to the final building. The result is a purpose build facility that should withstand the evolution of the technology and ever changing researcher needs 

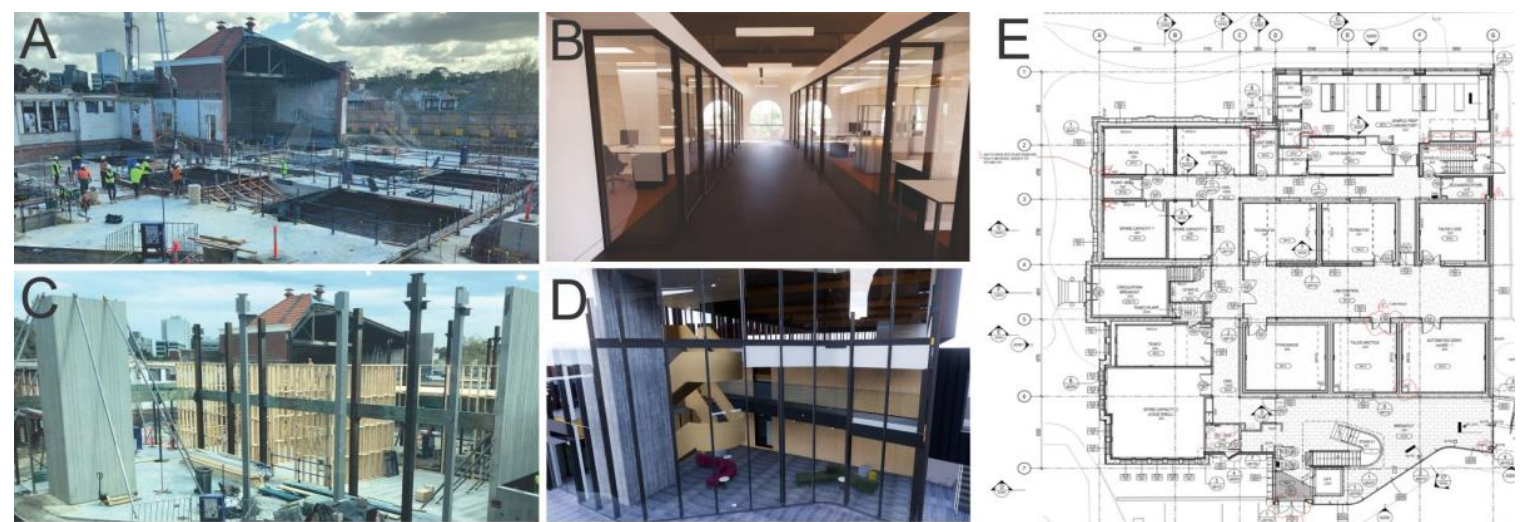

Figure 1. Construction site and concept design illustration. A) base inertia slabs and under roof office space B) office space C) building and microscope room frames D) atrium space E) architectural drawing of the ground floor. 